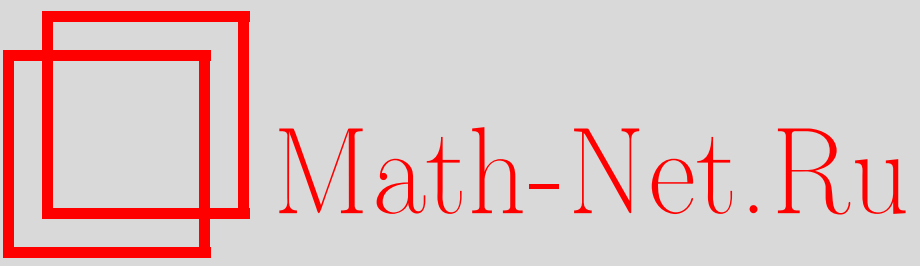

Ю. С. Попков, А. Ю. Попков, Ю. А. Дубнов, Кросс-энтропийная редукции матрицы данных с ограничением информационной емкости матриц-проекторов и их норм, Матем. моделирование, 2020, том 32, номер 9, 35-52

DOI: https://doi.org/10.20948/mm-2020-09-03

Использование Общероссийского математического портала Math-Net.Ru подразумевает, что вы прочитали и согласны с пользовательским соглашением

http: //www. mathnet.ru/rus/agreement

Параметры загрузки:

IP : 54.197 .217 .227

26 апреля 2023 г., 15:05:49 


\title{
КРОСС-ЭНТРОПИЙНАЯ РЕДУКЦИИ МАТРИЦЫ ДАННЫХ С ОГРАНИЧЕНИЕМ ИНФОРМАЦИОННОЙ ЕМКОСТИ МАТРИЦ-ПРОЕКТОРОВ И ИХ НОРМ
}

\author{
(C) 2020 2. $\quad$ Ю.С. Попков ${ }^{1,2}$, А.Ю. Попков ${ }^{1}$, Ю.А. Дубнов ${ }^{1,3}$ \\ ${ }^{1}$ Федеральный исследовательский центр «Информатика и управление» РАН, \\ Москва \\ 2 Институт проблем управления РАН, Москва \\ 3 Национальный исследовательский университет «Высшая школа экономики», \\ Москва \\ popkov@isa.ru; apopkov@isa.ru; yury.dubnov@phystech.edu
}

Работа выполнена при поддержке Российского фонда фундаментальных исследований (проекты 17-29-03119, 20-07-00470).

\section{DOI: $10.20948 / \mathrm{mm}-2020-09-03$}

Развивается метод сокращения размерности матрицы данных, основанный на ее прямом и обратном проектировании, и вычислении проекторов, минимизирующих кросс-энтропийный функционал. Вводится понятие информационной емкости матрицы, которое используется в качестве ограничения в задаче оптимальной редукции. Проводится сравнение предлагаемого метода с известными в задаче бинарной классификации.

Ключевые слова: понижение размерности, энтропия, кросс-энтропия, классификация.

\section{CROSS-ENTROPY REDUCTION OF DATA MATRIX WITH RESTRICTION ON INFORMATION CAPACITY OF PROJECTORS AND THEIR NORMS}

\author{
Y.S. Popkov ${ }^{1,2}$, A.Y. Popkov ${ }^{1}$, Y.A. Dubnov $v^{1,3}$ \\ ${ }^{1}$ Federal Research Center "Computer Science and Control" of RAS, Moscow \\ ${ }^{2}$ Institute of Control Sciences of RAS, Moscow \\ ${ }^{3}$ National Research University Higher School of Economics, Moscow
}

We develop a new method of dimensionality reduction based on direct and inverse projection of data matrix and calculation of projectors minimizing cross-entropy functional. Concept of information capacity of matrix which is used as a restriction in a problem of optimal reduction is introduced. We conduct a comparison of proposed method with known ones based on binary classification.

Keywords: dimensionality reduction, entropy, cross-entropy, classification. 


\section{1. Введение}

Задачи прогнозирования и классификации являются одними из наиболее распространенных в фундаментальных и прикладных исследованиях. Ядром этих задач является параметризованная модель (ПМ): динамическая - для прогнозирования и статическая - для классификации. ПМ «обучается» на ретроспективных данных, используя алгоритмы машинного обучения $[1,2]$, с последующим построением прогнозов или распределения объектов по классам.

Ретроспективные данные для указанных задач представляют собой матрицу, строки которой - объекты из обучающей коллекции данных, а столбцы - их признаки. Количество признаков $s$ определяет размерность признакового пространства и, соответственно, структуру ПМ. Количество объектов $m$ определяет объем обучающей коллекции.

При использовании $(m \times s)$-матриц данных возникает потребность сократить их размерность, т.е. аппроксимировать исходную матрицу размерности $(m \times s)$, эквивалентной в определенном смысле матрицей меньшей размерности $(n \times r)$, где $r<s, n<m$. Необходимость в такой редукции может быть связана как с природой объекта, некоторая данные о котором оказываются несущественными, взаимосвязанными или, напротив, случайными выбросами, так и по чисто техническим причинам. Например, - с имеющимися ограничениями ресурсов компьютера, где производятся вычисления.

Проблеме понижения размерности матрицы данных посвящено большое количество работ, например, $[3,4]$, среди которых можно выделить следующие три группы, различающиеся положенными в их основу идеями. В первой из них используется приближение заданного набора многомерных точек маломерным аффинным многообразием [5]. Наиболее распространенным является метод главных компонент (МГК) $[6,7]$ и его модификации $[8$, 9]. Опыт его применения в различных задачах показал его значительную чувствительность к выбросам в значениях данных. Поэтому одно из направлений его совершенствования связано с методами снижения этой чувствительности, т.е. придания ему робастных свойств [10]. Следует также отметить, что МГК представляет собой комбинацию математического метода получения упорядоченной последовательности собственных чисел матрицы данных и эвристических соображений по поводу «обрезания» этой последовательности. Кроме того его эффективность для задач заметной размерности остается предметом дискуссий.

Вторая группа работ базируется на так называемыми случайных проекuиях $[11,12]$. Основу их составляет лемма Джонсона-Линденштрауса [13] о 
сохранении в среднем расстояния между векторами исходного и редуцированного пространств. В [14] было показано, что для генерации матрицы со случайными элементами можно использовать стандартное нормальное распределение, а в некоторых случаях даже равномерное.

И наконец, в третью группу входят работы, использующие различные виды энтропийных функций для отбора наиболее информативных признаков. В частности, кросс-энтропия (или взаимная информация) позволяют установить наличие связей между признаками $[15,16]$.

В данной работе, которую следует отнести к «энтропийной» группе, развивается метод энтропийного понижения размерности EDR (Entropy Dimensionality Reduction). Вводится понятие информационной емкости матрицы данных и матриц-проекторов, которые характеризуются своими фробениусовыми нормами.

Предлагаемый EDR-метод, в отличии от DIP-метода [17], позволяет формировать энтропийно-оптимальные матрицы-проекторы при ограничениях на нормы матриц-проекторов и допустимые информационной емкости матриц-проекторов.

Редуцированная матрица данных используется для обучения предсказательной модели, применяемой для решения задач прогнозирования и классификации. Поэтому важным является характеризация ошибок, возникающих в указанных задачах при использовании редуцированных матриц данных вместо исходных. Приводятся также результаты вычислительных экспериментов, связанных с сравнением последствий (ошибок) различных методов редукции при решении задач линейной бинарной классификации.

\section{2. Характеристики кросс-энтропийной редукции размерности}

1. «Прямое» и «обратное» проектирование Рассмотрим матрицу данных $U$ размерности $(m \times s)$ с неотрицательными элементами, где $m$ - количество объектов, $s$ - количество признаков, характеризующих каждый объект. Пусть размерность $r<s$ желаемого количества признаков задана. Введем матрицу $Q=\left[q_{i j} \geq 0, i=\overline{1, s}, j=\overline{1, r}\right]^{1}$. Осуществим операцию проектирования («прямого») на подпространство $R_{+}^{(m r)}$ с помощью матрицы $Q_{(s \times r)}$ :

$$
Y_{(m \times r)}=U_{(m \times s)} Q_{(s \times r)} .
$$

\footnotetext{
${ }^{1}$ Неотрицательность матрицы $Q$, вообще говоря, не требуется для дальнейшего, но существенно упрощает задачу проектирования
} 
Матрица $Y$ состоит из $m$ векторов-строк, размером $(r<s)$. Осуществим операцию проектирования («обратного») на пространство $R_{+}^{(m s)}$ (исходных данных) с помощью матрицы $T_{(r \times s)}$ с неотрицательными элементами:

$$
X_{(m \times s)}=U_{(m \times s)} Q_{(s \times r)} T_{(r \times s)} .
$$

Матрица $X_{(m \times s)}$ имеет ту же размерность, что и исходная матрица данных $U_{(m \times s)}$, и неотрицательные элементы.

2. Кросс-энтропийное «расстояние». Для характеризации «расстояния» между матрицами $X$ и $U$ определим функиионал кросс-энтропии $H(X \mid U)$ при фиксированной матрице $U$ в виде:

$$
\begin{aligned}
& H\left(X_{(m \times s)} \mid U_{(m \times s)}\right)=H\left(Q_{(s \times r)}, T_{(r \times s)} \mid U\right)= \\
& \quad=-\operatorname{tr}\left[X(Q, T) L^{\mathrm{T}}(Q, T)\right]=\operatorname{tr}\left[L(Q, T) X^{\mathrm{T}}(Q, T)\right],
\end{aligned}
$$

где

- $L(Q, T)$ - матрица с элементами $\ln \left(x_{i j}(Q, T) / u_{i j}\right)$;

- $\operatorname{tr}(\bullet)$ - след произведения указанных матриц;

- элементы матрицы $X$ связаны с элементами матриц $Q, T$ следующими соотношениями:

$$
x_{i j}\left(Q_{(s \times r)}, T_{(r \times s)} \mid U_{(m \times s)}\right)=\sum_{\mu=1}^{r} \sum_{v=1}^{s} t_{\mu, j} q_{v, \mu} u_{i, v}, \quad i=\overline{1, m} ; j=\overline{1, s} .
$$

Компоненты градиента функции $H(Q, T \mid U)$ по элементам матриц $Q, T$ имеют следующий вид:

$$
\begin{aligned}
& \nabla_{v, \mu}^{Q}=\frac{\partial H(Q, T \mid U)}{\partial q_{v, \mu}}=\sum_{i=1}^{m} \sum_{j=1}^{s}\left[\ln \frac{x_{i j}}{u_{i j}}+1\right] t_{\mu, j} u_{i, v}, \quad v=\overline{1, s}, \mu=\overline{1, r}, \\
& \nabla_{\mu, h}^{T}=\frac{\partial H(Q, T \mid U)}{\partial t_{\mu, h}}=-\sum_{i=1}^{s} \sum_{j=1}^{m}\left[\ln \frac{x_{i j}}{u_{i j}}+1\right] \sum_{v=1}^{s} q_{v, \mu} u_{i, v}, \quad \mu=\overline{1, r}, h=\overline{1, s} .
\end{aligned}
$$

Приближенная кросс-энтропия. Иногда может оказаться полезным приближенное выражение $H$-функционала (3), которое можно получить, аппроксимируя логарифмические функции в (3) в окрестности точек $x_{i j}=u_{i j}$

$$
\ln \frac{x_{i j}}{u_{i j}} \simeq \frac{1}{u_{i j}}\left(x_{i j}-u_{i j}\right)<\frac{1}{u_{\min }}\left(x_{i j}-u_{i j}\right) .
$$


Кросс-энтропийная редукиии матрицы данных с ограничением ...

Применяя эту аппроксимацию, получим следующее приближенное выражение:

$$
\begin{aligned}
& H(Q, T \mid U) \approx=\operatorname{tr}\left(X X^{\mathrm{T}}\right)-\operatorname{tr}\left(X U^{\mathrm{T}}\right)= \\
& \quad=(X(Q, T), X(Q, T))-(X(Q, T), U)=\tilde{H}(Q, T \mid U) .
\end{aligned}
$$

где $(A, B)$ - фробениусово скалярное произведение [18],

$$
\operatorname{tr}\left(A B^{\mathrm{T}}\right)=\operatorname{tr}\left(B A^{\mathrm{T}}\right)=(A, B)=(B, A) .
$$

Используя правила матричного дифференцирования [18], получим следующие выражения для градиентов $\Delta_{Q}(Q, T), \Delta_{T}(Q, T)$ функционала $\tilde{H}(Q, T \mid U)$ (8) по матрицам $Q, T$ :

$$
\begin{aligned}
& \Delta_{Q}(Q, T)=\frac{\partial \tilde{H}(Q, T \mid U)}{\partial X} \frac{\partial X}{\partial Q}=2 T P Q T-T P . \\
& \Delta_{T}(Q, T)=\frac{\partial \tilde{H}(Q, T \mid U)}{\partial X} \frac{\partial X}{\partial T}=2 Q^{\mathrm{T}} P Q T-Q^{\mathrm{T}} P .
\end{aligned}
$$

Здесь $P=X X^{\mathrm{T}}$. Градиенты функции $\tilde{H}(Q, T \mid U)$ по матрицам $Q$ и $T$ представляет собой матрицы размером $(s \times r)$.

Напомним правила дифференцирования некоторых скалярных функций, зависящих от матриц [18]. Рассмотрим линейное пространство $(m \times n)$ матриц $A$. Скалярное произведение в этом пространстве:

$$
(A, B)=\operatorname{tr}\left(A B^{\mathrm{T}}\right)=\operatorname{tr}\left(B A^{\mathrm{T}}\right) .
$$

Норма (фробениусова): $\|A\|^{2}=(A, A)$.

Из этих определений видно, что матрица рассматривается как вектор, составленный из примыкающих строк (векторизация матрицы). Для скалярной функции от матрицы градиент определяется следующим образом. Рассмотрим скалярную функцию $f(A)$. Ее приращение представимо в виде:

$$
f(A+\Delta)=f(A)+(H, \Delta)+(\Delta) .
$$

Здесь $(\Delta)$ - остаток ряда Тейлора. Градиент функции $f(A)$ по матрице $A$

$$
\Delta_{A} f(A)=H .
$$

Приведем простые, но полезные примеры скалярных функций от матрицы:

$$
f(A)=(C, A), \quad \Delta_{A} f(A)=C .
$$




$$
f(A)=\|A\|^{2}, \quad \Delta_{A} f(A)=2 A .
$$

Функционалы $H(Q, T \mid U)$ и $\tilde{H}(Q, T \mid U)$ являются характеристиками «расстояния» между матрицами $X$ и $U$.

3. Фробениусовы нормы матриц. Выше предполагалось, что матрицы прямого $Q$ и обратного проектирования $T$ имеют неотрицательные элементы. Последнее связано с применением кросс-энтропии для характеризации «расстояния» между ними. Однако, часто, в прикладных задачах машинного обучения имеет смысл класс матриц-проекторов сузить. Этого можно достичь, используя фробениусову матричную норму:

$$
\begin{aligned}
& n_{Q}(Q)=\sum_{i=1}^{s} \sum_{j=1}^{r} q_{i j}^{2}=\operatorname{tr}\left(Q Q^{\mathrm{T}}\right)=(Q, Q) ; \\
& n_{T}(T)=\sum_{i=1}^{r} \sum_{j=1}^{s} t_{i j}^{2}=\operatorname{tr}\left(T T^{\mathrm{T}}\right)=(T, T) .
\end{aligned}
$$

4. Информационная емкость матрицы. В процессе редукции матрицы данных, информация, которая в ней заключена частично теряется. Поэтому ее нужно как-то измерять, а затем строить так процедуру редукции, чтобы потери были бы минимальными или ограниченными.

Определим информационную емкость $\mathcal{I}(A)$ матрицы $A_{n \times q}$ с неотрицательными элементами в виде:

$$
\mathcal{I}(A)=\sum_{i=1}^{n} \sum_{j=1}^{q} a_{i j} \ln a_{i j} .
$$

\section{3. Алгоритм кросс-энтропийной редукции}

Процедура редукции матрицы данных сводится к минимизации кроссэнтропии (3):

$$
H(Q, T \mid U) \Rightarrow \min _{Q, T}
$$

где $H(Q, T \mid U)=\sum_{i=1}^{m} \sum_{j=1}^{s} x_{i j}(Q, T \mid U) \ln \frac{x_{i j}(Q, T \mid U)}{u_{i j}}$,

$$
x_{i j}(Q, T \mid U)=\sum_{\mu=1}^{r} \sum_{v=1}^{s} t_{\mu, j} q_{v, \mu} u_{i, v}, \quad i=\overline{1, m} ; j=\overline{1, s} .
$$

при ограничениях:

- неотрицательности матриц-проекторов 
Кросс-энтропийная редукции матрицьы данных с ограничением ...

$Q \geq 0, \quad T \geq 0 ;$

- $(a, b)$-нормы матриц-проекторов (12)

$n_{Q}(Q)=\sum_{i=1}^{s} \sum_{j=1}^{r} q_{i j}^{2} \leq a, \quad n_{T}(T)=\sum_{i=1}^{r} \sum_{j=1}^{s} t_{i j}^{2} \leq b$

- $\delta$-допустимого снижения информационной емкости

$$
\frac{\mathcal{I}(U)-\mathcal{I}(Y(Q \mid U))}{\mathcal{I}(U)} \leq \delta
$$

В неравенстве (19)

$$
\begin{aligned}
& \mathcal{I}(Y(Q \mid U))=\sum_{i=1}^{m} \sum_{j=1}^{r} y_{i j}(Q \mid U) \ln y_{i j}(Q \mid U) . \\
& y_{i j}(Q \mid U)=\sum_{v=1}^{S} u_{i, v} q_{v, j} .
\end{aligned}
$$

В силу свойств целевой функции (энтропийно-билинейная форма), ограничений в виде квадратичных энтропийных неравенств, задача кросс-энтропийной редукции (14)-(18) является невыпуклой $[19,20]$. Поэтому в ней существует некоторое количество (неизвестное заранее) локальных экстремумов, и указанную задачу следует рассматривать как задачу поиска глобального экстремума.

Здесь ограничимся рассмотрением задачи (14)-(18) как задачи локальной минимизации на неотрицательном ортанте $R_{+}^{2 s r}$. Переменными в этой задаче являются элементы матриц $Q, T$, в каждой $r s$ элементов. Используя векторизацию матриц, т.е. переход к вектору путем последовательного примыкания строк, сформируем векторы $\mathbf{q}, \mathbf{t}, \mathbf{u}$, соответствующие матрицам $Q, T, U$. Тогда задача минимизации кросс-энтропии может быть представлена в виде:

$$
\begin{aligned}
& \mathcal{H}(\mathbf{q}, \mathbf{t} \mid \mathbf{u}) \Rightarrow \min , \\
& (\mathbf{q}, \mathbf{t}) \in R_{+}^{(2 s r)}, \\
& N_{Q}(\mathbf{q})=-\tilde{N}_{Q}(\mathbf{q})+a \geq 0, \quad N_{T}(\mathbf{t})=-\tilde{N}_{T}(\mathbf{t})+b \geq 0, \\
& \mathcal{I}_{Y}(\mathbf{q})=I_{Y}(\mathbf{q} \mid \mathbf{u})-\gamma(\delta) \geq 0, \quad \gamma(\delta)=(1-\delta) I_{U} .
\end{aligned}
$$

Напомним, что задача (22) рассматривается как задача локальной минимизации, для которой условия Куна-Таккера являются необходимыми. Функция Лагранжа для данной задачи имеет вид 


$$
L(q, t, \alpha, \beta, \gamma)=\mathcal{L}(q, t, \gamma)-\langle\alpha, q\rangle-\langle\beta, t\rangle,
$$

где $\alpha=\left\{\alpha_{1}, \ldots, \alpha_{r s}\right\}, \beta=\left\{\beta_{1}, \ldots, \beta_{r s}\right\}$ и $\gamma=\left\{\gamma_{1}, \gamma_{2}, \gamma_{3}\right\}-$ множители Лагранжа при соответствующих ограничениях; функцию

$$
\mathcal{L}(\mathbf{q}, \mathbf{t}, \gamma)=\mathcal{H}(\mathbf{q}, \mathbf{t})-\gamma_{1} N_{Q}(\mathbf{q})-\gamma_{2} N_{T}(\mathbf{t})-\gamma_{3} \mathcal{I}_{Y}(\mathbf{q})
$$

будем называть усеченной функцией Лагранжа.

Пусть существует точка $\left(\mathbf{q}^{*}, \mathbf{t}^{*}\right)$ локального минимума в задаче $(22,24)$ и окрестность

$$
\mathcal{O}_{\left(\mathbf{q}^{*}, \mathbf{t}^{*}\right)}(\mathbf{q}, \mathbf{t}) \in\left\|\mathbf{q}^{*}-\mathbf{q}\right\|^{2}+\left\|\mathbf{t}^{*}-\mathbf{t}\right\|^{2} \leq \rho
$$

такая, что на множестве (26) задача (22), (24) выпукла. Тогда на множестве (26) выполняются необходимые условия Куна-Таккера [19] в терминах усеченной функции Лагранжа (25):

$$
\begin{aligned}
& \nabla_{\mathbf{q}} \mathcal{L}(\mathbf{q}, \mathbf{t}, \gamma) \geq 0, \quad \nabla_{\mathbf{t}} \mathcal{L}(\mathbf{q}, \mathbf{t}, \gamma) \geq 0, \\
& \mathbf{q} \otimes \nabla_{\mathbf{q}} \mathcal{L}(\mathbf{q}, \mathbf{t}, \gamma) \geq 0, \quad \mathbf{t} \otimes \nabla_{\mathbf{t}} \mathcal{L}(\mathbf{q}, \mathbf{t}, \gamma) \geq 0, \\
& \mathbf{q}^{*} \geq 0, \quad \mathbf{t}^{*} \geq 0, \\
& \nabla_{\gamma} \mathcal{L}(\mathbf{q}, \mathbf{t}, \gamma) \leq 0, \quad \gamma \otimes \nabla_{\gamma} \mathcal{L}(\mathbf{q}, \mathbf{t}, \gamma)=0, \quad \gamma \geq 0 .
\end{aligned}
$$

Для решения этой системы уравнений и неравенств можно применить мультипликативный алгоритм [21]. Введем следующие обозначения:

$$
\begin{aligned}
& \mathbf{G}_{\mathbf{q}}(\mathbf{q}, \mathbf{t}, \gamma)=1-\theta \nabla_{\mathbf{q}} \mathcal{L}(\mathbf{q}, \mathbf{t}, \gamma), \\
& \mathbf{G}_{\mathbf{t}}(\mathbf{q}, \mathbf{t}, \gamma)=1-\theta \nabla_{\mathbf{t}} \mathcal{L}(\mathbf{q}, \mathbf{t}, \gamma), \\
& \mathbf{K}_{\gamma}(\mathbf{q}, \mathbf{t}, \gamma)=1+\theta \nabla_{\gamma} \mathcal{L}(\mathbf{q}, \mathbf{t}, \gamma) .
\end{aligned}
$$

Мультипликативный алгоритм Minimum Cross-Entropy with constraints (МСЕС) имеет вид:

- начальный шаг

$$
\mathbf{q}^{0}>0, \quad \mathbf{t}^{0}>0
$$

- $n$-й итерационный шаг

$$
\begin{aligned}
& \mathbf{q}^{(n+1)}=\mathbf{q}^{n} \otimes \mathbf{G}_{\mathbf{q}}\left(\mathbf{q}^{n}, \mathbf{t}^{n}, \gamma^{n}\right), \quad \mathbf{t}^{(n+1)}=\mathbf{t}^{n} \otimes \mathbf{G}_{\mathbf{t}}\left(\mathbf{q}^{n}, \mathbf{t}^{n}, \gamma^{n}\right) ; \\
& \mathbf{t}^{(n+1)} \rightarrow T^{(n+1)} ; \quad \gamma^{n+1}=\gamma^{n} \otimes \mathbf{K}_{\gamma}\left(\mathbf{q}^{n}, \mathbf{t}^{n}, \gamma^{n}\right) ;
\end{aligned}
$$


Кросс-энтропийная редукции матрицьы данных с ограничением ...

$$
\begin{aligned}
& \mathbf{q}^{(n+1)} \rightarrow Q^{(n+1)} ; \quad \mathbf{t}^{(n+1)} \rightarrow T^{(n+1)} ; \quad X^{(n+1)}=U Q^{(n+1)} T^{(n+1)} ; \\
& H\left(Q^{(n+1)}, T^{(n+1)} \mid U\right)=\sum_{i=1}^{m} \sum_{j=1}^{r} x_{i j}^{(n+1)} \ln \frac{x_{i j}^{(n+1)}}{u_{i j}}=H^{(n+1)} . \\
& - \text { условие остановки } \\
& H^{(n+1)}-H^{(n)} \leq \Delta .
\end{aligned}
$$

Данный алгоритм сходится к локальному экстремуму-минимуму при начальной точке с положительными координатами. Доказательство глобальной сходимости для задач выпуклового программирования приведено в [21]. В случае невыпуклых задач имеет место локальная сходимость к какому-нибудь локальному экстремуму, попадание в который зависит от начального приближения. Схема доказательства приведена в приложении.

\section{4. Оценивание эффективности метода для задач бинарной классификации}

Характеризацию эффективности энтропийного метода EDR и сравнение его с методом главных компонент (МГК) и методом случайных проекций (МСП) будем проводить с помощью вычислительного эксперимента с использованием реальных данных для задачи бинарной классификации, в которой применяется линейный классификатор.

Напомним некоторые сведения, связанные с задачей линейной классификации $[2,22,23]$. Модель классификатора имеет вид:

$$
z\left(t_{k}\right)=\operatorname{sign}\left(\sum_{i=1}^{s} a_{i} u_{i}\left(t_{k}\right)\right) .
$$

Параметры $\mathbf{a} \in R^{S}$ - веса признаков, неслучайные, но значения их неизвестны. Функция

$$
\operatorname{sign}(x)=\left(\begin{array}{ll}
+1, & \sum_{i=1}^{S} a_{i} u_{i}\left(t_{k}\right) \geq 0, \\
-1, & \sum_{i=1}^{S} a_{i} u_{i}\left(t_{k}\right)<0 .
\end{array}\right.
$$

Имеется коллекция обучающих данных $\mathcal{L}\left(\mathbf{y}, U_{(m \times s)}\right)$. Вектор $\mathbf{y} \in R^{m}$, а его компоненты имеют значение +1 , если объект с номером $t_{k}$ принадлежит первому классу, и -1 , если объект с номером $t_{k}$ принадлежит второму классу. Строки матрицы $U_{m \times s}$ содержат значения признаков $t_{k}$-объектов, где $k=\overline{1, m}$. Обучение классификатора (29) минимизации эмпирического риска 


$$
J(\mathbf{a})=\sum_{i=1}^{m}\left\|\mathbf{y}-\mathbf{z}\left(\mathbf{a} \mid U_{(m \times s)}\right)\right\|^{2}
$$

по параметрам а. Здесь компоненты вектора $\mathbf{z}$ генерируются моделью классификатора (29).

Для тестирования обученного классификатора используется другая коллекция данных $\mathcal{T}=\left(\mathbf{x}, V_{(n \times s)}\right)$, где вектор $\mathbf{x} \in R^{n}$. Классификатор

$$
w\left(t_{k}\right)=\operatorname{sign}\left(\sum_{i=1}^{s} \hat{a}_{i} v_{i}\left(t_{k}\right)\right), \quad k=\overline{1, n}
$$

выдает $w\left(t_{k}\right)=+1$ или $w\left(t_{k}\right)=-1$ по предъявлению строк матрицы $V_{(n \times s)}$. Они сравниваются с тестовым вектором $\mathbf{x}$ и подсчитывается количество совпадений (правильная классификация), т.е.

$$
I=\sum_{k=1}^{n} \Lambda\left(w\left(t_{k}\right)-x\left(t_{k}\right)\right)
$$

где характеристическая функция

$$
\Lambda(y)=\left(\begin{array}{lll}
1, & \text { если } & y=0, \\
0, & \text { если } & y \neq 0 .
\end{array}\right.
$$

Тогда точность (accuracy) $\varepsilon$ классификации характеризуется отношением

$$
\varepsilon=I / n \text {. }
$$

\section{5. Схема вычислительного эксперимента}

Экспериментальное исследование метода EDR, следуя [24, 25], проводится на основе решения задачи бинарной классификации в исходном и редуцированных пространствах признаков с последующим сравнением показателей точности (35) классификации для энтропийного метода (EDR), метода главных компонент (PCA) и метода случайных проекций (RP). Для обеспечения стабильности получаемых результатов применялся метод Монте-Карло и перекрестная проверка (кросс-валидация).

Эксперимент состоит из нескольких этапов:

1. Решение задачи классификации в исходном пространстве;

2. Решение задачи классификации в редуцированных по РСА пространствах;

3. Решение задачи классификации в редуцированных по RP пространствах;

4. Решение задачи классификации в редуцированных по EDR пространствах. 
Этапы 2-4 последовательно реализуются для сниженной на единицу размерности, то есть эти этапы реализуются для $r=s-1, \ldots, 1$, где $r-$ размерность редуцированных пространств. Этап 4 реализуется для различных значений $\delta$ (допустимое снижение информационной емкости).

Программное обеспечение для проведения экспериментального исследования было реализовано на платформе Python в среде Linux с использованием библиотек numpy 1.14.3, scipy 1.1.0, sklearn 0.19.1. Сокращение размерности методами главных компонент и случайных проекций проводилось с помощью соответствующих классов библиотеки scikit-learn [26]: PCA и GaussianRandomProjection, решение задачи классификации методом опорных векторов [1] - с помощью класса SVC с линейным ядром.

В экспериментальном исследовании использовались два набора данных. Первый набор simulation является синтетическим набором данных из 100 объектов с 10 признаками. Данные генерируются функцией make_classification [27] с параметрами: n_classes $=2$, n_clusters_per_class $=2, n$ _informative $=$ $=10, \mathrm{n} \_$redundant $=0$, class_sep $=1.0$. Эта функция генерирует точки в пространстве размерности $n \_$informative, нормально распределенные (с нулевым математическим ожиданинем и единичной дисперсией) вокруг ребер гиперкуба со сторонами 2 * class_sep с добавлением некоторого шума.

Второй набор данных heart представляет из себя реальные данные, собранные в рамках исследований болезней сердца [28]. Набор содержит 270 объектов из 13 признаков.

Во всех экспериментах данные масштабируются на интервал $[0,1]$.

\section{6. Результаты экспериментов}

Во всех экспериментах использовалась кросс-валидация по методу bootstrap [1], состоящая в следующем. Из исходного набора данных 2 раза случайно выбирались 30 объектов, 20 из которых использовались для обучения классификатора, остальные 10 использовались для тестирования, результаты по каждой выборке осреднялись. Далее производилось 10 повторений эксперимента, результаты по которым также осреднялись. Снижение размерности на каждом этапе проводилось для всей обучающей выборки (30 объектов).

Для решения задачи (14)-(19) был использован метод условной оптимизации на неотрицательном ортанте SLSQP [29], реализованный в библиотеке scipy функцией minimize с параметрами: ftol=1e-4, maxiter=1000. Необходимо отметить, что при некоторых значениях $\delta$ метод не мог найти решение при заданных параметрах оптимизации. В этих случаях использовалось последнее найденное им решение. 
1. Зависимость точности є от количества объектов (объем выборки т). Исследование этой зависимости для трех методов редукции важно для выявления областей их применения. Известно, что методы максимизации энтропии и производные от них, в частности, EDR-метод, обычно применяются при ограниченном объеме данных по сравнению с размерностью признакового пространства. При «больших данных» принципиальных ограничений по их применению нет, но существенно возрастают вычислительные трудности. Что касается МГК, то известно, что при больших объемах данных ( $m$ достаточно велико) и их неоднородности метод становится неустойчивым [10].

Таблица 1. Точность классификации набора данных simulation с редукцией размерности методом РСА для разного объема обучающей выборки $m$.

\begin{tabular}{|c|c|c|c|c|c|c|}
\hline$m-r$ & 10 & 9 & 8 & 7 & 6 & 5 \\
\hline 10 & 0.60 & 0.60 & 0.60 & 0.60 & 0.67 & 0.67 \\
\hline 9 & 0.48 & 0.48 & 0.48 & 0.48 & 0.48 & 0.52 \\
\hline 8 & 0.46 & & 0.46 & 0.46 & 0.46 & 0.46 \\
\hline 7 & 0.48 & & & 0.48 & 0.48 & 0.48 \\
\hline 6 & 0.44 & & & & 0.44 & 0.44 \\
\hline 5 & 0.40 & & & & & 0.40 \\
\hline
\end{tabular}

Таблица 2. Точность классификации набора данных simulation с редукцией размерности методом RP для разного объема обучающей выборки $m$.

\begin{tabular}{|c|c|c|c|c|c|c|}
\hline$m r$ & 10 & 9 & 8 & 7 & 6 & 5 \\
\hline 10 & 0.60 & 0.67 & 0.57 & 0.77 & 0.60 & 0.57 \\
\hline 9 & 0.48 & 0.48 & & & & \\
\hline 9 & 0.48 & 0.48 & 0.59 & 0.41 & 0.52 & 0.48 \\
\hline 8 & 0.46 & 0.46 & 0.46 & 0.46 & 0.46 & 0.54 \\
\hline 7 & 0.48 & 0.48 & 0.52 & 0.38 & 0.43 & 0.52 \\
\hline 6 & 0.44 & 0.28 & 0.39 & 0.39 & 0.39 & 0.39 \\
\hline 5 & 0.40 & 0.40 & 0.40 & 0.40 & 0.40 & 0.40 \\
\hline
\end{tabular}

Таблица 3. Точность классификации набора данных simulation с редукцией размерности методом EDR для разного объема обучающей выборки $m$ при $\delta=0.5$.

\begin{tabular}{|c|c|c|c|c|c|c|}
\hline$m-r$ & 10 & 9 & 8 & 7 & 6 & 5 \\
\hline 10 & 0.60 & 0.70 & 0.70 & 0.63 & 0.50 & 0.53 \\
\hline 9 & 0.48 & 0.37 & 0.52 & 0.59 & 0.37 & 0.37 \\
\hline 8 & 0.46 & 0.46 & 0.46 & 0.46 & 0.46 & 0.50 \\
\hline 7 & 0.48 & 0.43 & 0.43 & 0.48 & 0.48 & 0.57 \\
\hline 6 & 0.44 & 0.44 & 0.28 & 0.39 & 0.44 & 0.39 \\
\hline 5 & 0.40 & 0.53 & 0.47 & 0.53 & 0.60 & 0.40 \\
\hline
\end{tabular}

Было проведено исследование зависимости точности классификации от малых объемов выборки для набора данных simulation. Результаты приведены в табл.1-3 для $m=\overline{5,10}$. Для каждого из этих наборов проводилась редукция признакового пространства до размера $r=\overline{5,10}$. 
Кросс-энтропийная редукиии матрицы данных с ограничением ...

Из табл.1-3 видно, что методы EDR и RP демонстрируют работоспособность при любых соотношениях объемов выборки и размерностей признакового пространства, метод РСА при $m<r-$ не работает.

2. Точность классификачии при $m \gg r$. В табл.4, 5 и на рис. 1 представлены значения точности классификации для различной размерности редуцированного пространства. Оценка точности классификации проводилась для двух наборов данных - simulation и heart, чтобы исследовать устойчивость оценок по отношению к наборам данных.

Таблица 4. Точность классификации набора данных simulation.

\begin{tabular}{|c|c|c|c|c|c|c|}
\hline$r$ & 10 & 9 & 8 & 7 & 6 & 5 \\
\hline PCA & 0.65 & 0.64 & 0.63 & 0.65 & 0.65 & 0.64 \\
\hline RP & 0.65 & 0.58 & 0.54 & 0.58 & 0.62 & 0.48 \\
\hline EDR & 0.65 & 0.62 & 0.62 & 0.47 & 0.48 & 0.49 \\
\hline
\end{tabular}

Таблица 5. Точность классификации набора данных heart.

\begin{tabular}{|c|c|c|c|c|c|c|}
\hline$r$ & 13 & 12 & 11 & 10 & 9 & 8 \\
\hline PCA & 0.76 & 0.76 & 0.76 & 0.76 & 0.76 & 0.76 \\
\hline RP & 0.76 & 0.79 & 0.79 & 0.73 & 0.76 & 0.78 \\
\hline EDR & 0.76 & 0.70 & 0.78 & 0.74 & 0.76 & 0.69 \\
\hline
\end{tabular}
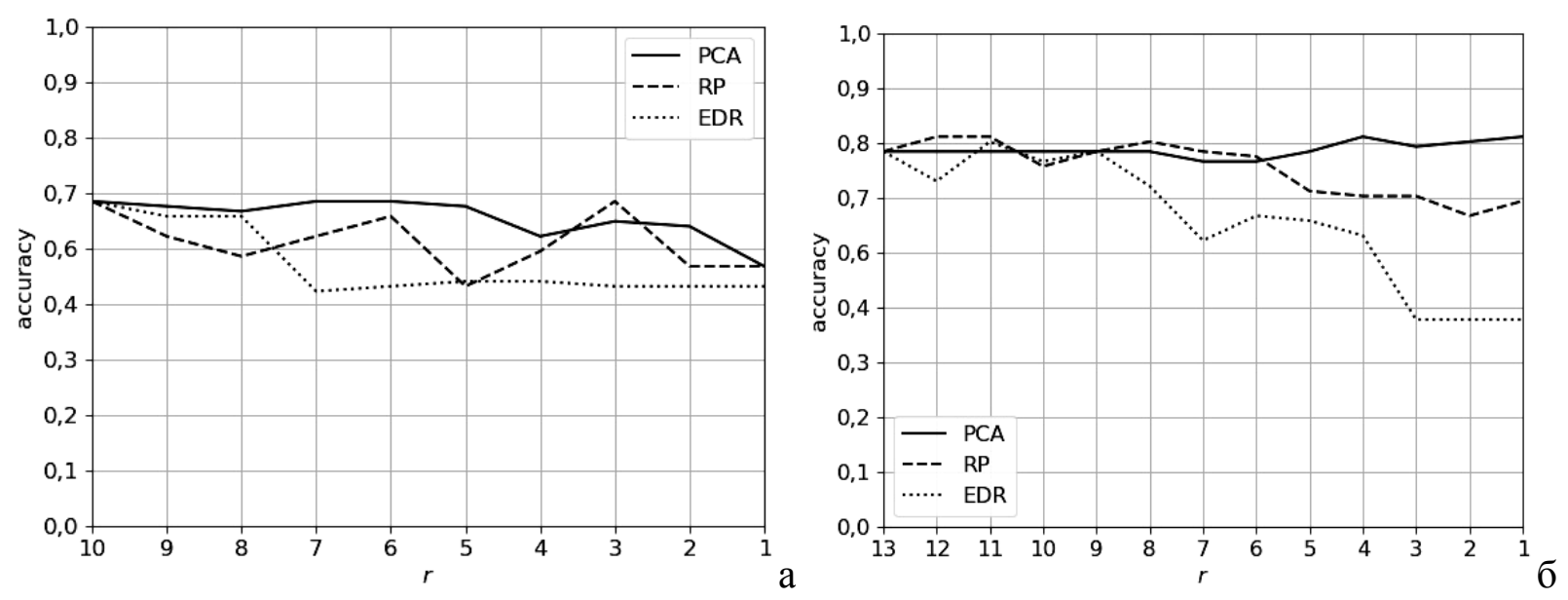

Рис.1. Точность классификации для наборов данных simulation и heart.

3. Зависимость точности \& EDR-метода от допустимого $\delta$ снижения информационной емкости. В табл.6 представлены значения точности классификации для различных значений $\delta$ и на рис. 2 соответствующие им графики на наборе данных simulation. В табл.7 представлены значения точности классификации для различных значений $\delta$ и на рис.2 соответствующие им графики на наборе данных heart. 
Таблица 6. Зависимость $\varepsilon(\delta)$ для набора данных simulation (EDR-метод).

\begin{tabular}{|c|c|c|c|c|c|}
\hline$\delta$ & 9 & 8 & 7 & 6 & 5 \\
\hline 0.1 & 0.60 & 0.65 & 0.50 & 0.50 & 0.50 \\
\hline 0.2 & 0.70 & 0.60 & 0.55 & 0.45 & 0.45 \\
\hline 0.3 & 0.75 & 0.75 & 0.40 & 0.45 & 0.50 \\
\hline 0.4 & 0.55 & 0.50 & 0.45 & 0.50 & 0.55 \\
\hline 0.5 & 0.50 & 0.60 & 0.45 & 0.50 & 0.45 \\
\hline
\end{tabular}

Таблица 7. Зависимость $\varepsilon(\delta)$ для набора данных heart (EDR-метод).

\begin{tabular}{|c|c|c|c|c|c|}
\hline$\delta$ & 12 & 11 & 10 & 9 & 8 \\
\hline 0.1 & 0.65 & 0.85 & 0.80 & 0.80 & 0.55 \\
\hline 0.2 & 0.60 & 0.70 & 0.70 & 0.60 & 0.65 \\
\hline 0.3 & 0.75 & 0.75 & 0.75 & 0.80 & 0.75 \\
\hline 0.4 & 0.75 & 0.85 & 0.80 & 0.80 & 0.70 \\
\hline 0.5 & 0.75 & 0.75 & 0.65 & 0.80 & 0.80 \\
\hline
\end{tabular}
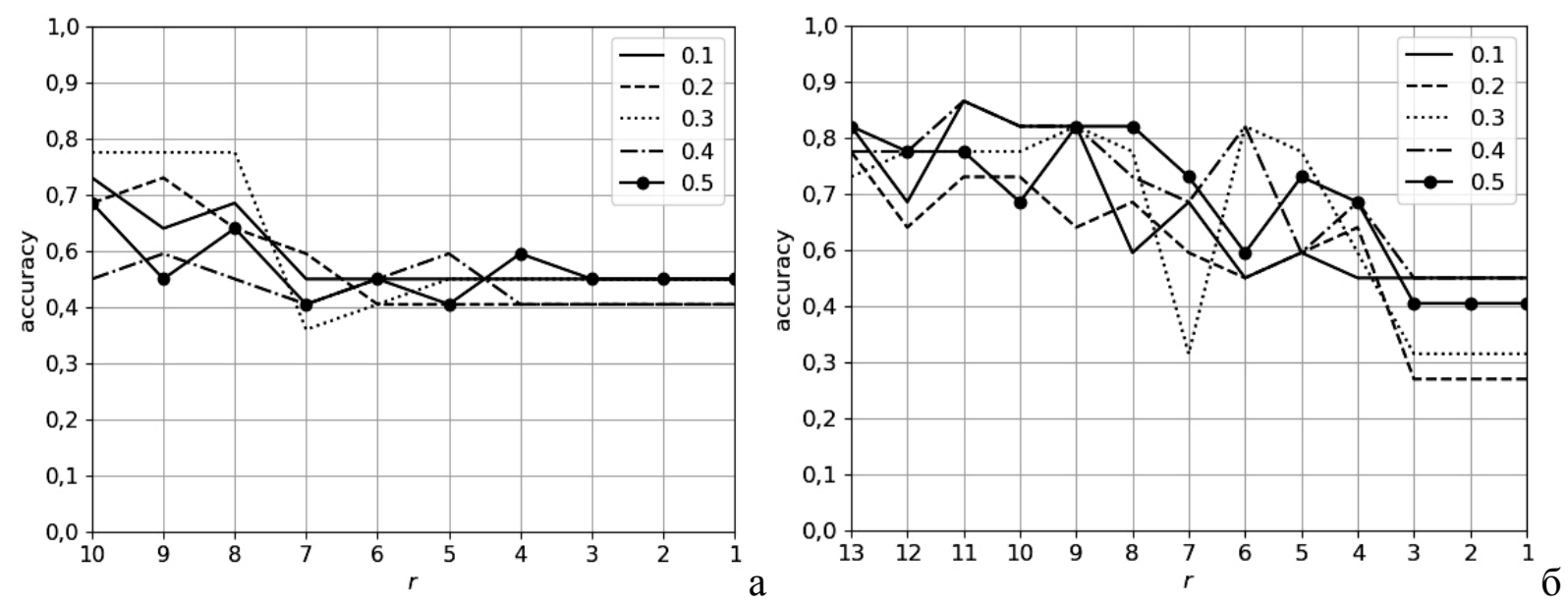

Рис.2. Зависимость $\varepsilon(\delta)$ для наборов данных simulation и heart (EDR-метод).

4. Информационные потери при редукции. Выше отмечалось, что при реукции признакового пространства возникают информационные потери. Согласно (21) относительная величина информационных потерь определяется следующим равенством:

$$
I=\frac{\mathcal{I}(U)-\mathcal{I}(Y)}{\mathcal{I}(U)},
$$

где $Y$ - редуцированная матрица данных. Исследовались информационные потери, возникающие при редукции методами PCA, RP и EDR на наборах данных simulation и heart.

В табл.8-9 представлены значения относительных информационных потерь I (36). Для метода EDR указаны средние по $\delta$ значения. 
Кросс-энтропийная редукции матрицьь данных с ограничением ...

Таблица 8. Относительные информационные потери для набора данных simulation.

\begin{tabular}{|c|c|c|c|c|c|}
\hline$r$ & 9 & 8 & 7 & 6 & 5 \\
\hline PCA & 0.6691 & 0.6979 & 0.7298 & 0.7616 & 0.7973 \\
\hline RP & 0.6630 & 0.6818 & 0.7844 & 0.7618 & 0.8777 \\
\hline EDR & 0.2688 & 0.2911 & 0.3034 & 0.3501 & 0.4125 \\
\hline
\end{tabular}

Таблица 9. Относительные информационные потери для набора данных heart.

\begin{tabular}{|c|c|c|c|c|c|c|}
\hline$r$ & 12 & 11 & 10 & 9 & 8 & 7 \\
\hline PCA & 0.2935 & 0.3317 & 0.3857 & 0.4399 & 0.4941 & 0.5671 \\
\hline RP & 0.1444 & 0.3708 & 0.5190 & 0.6051 & 0.6736 & 0.8167 \\
\hline EDR & 0.3083 & 0.3013 & 0.3184 & 0.2772 & 0.2589 & 0.2792 \\
\hline
\end{tabular}

На рис.3 изображены графики относительных информационных потерь для наборов данных simulation и heart.
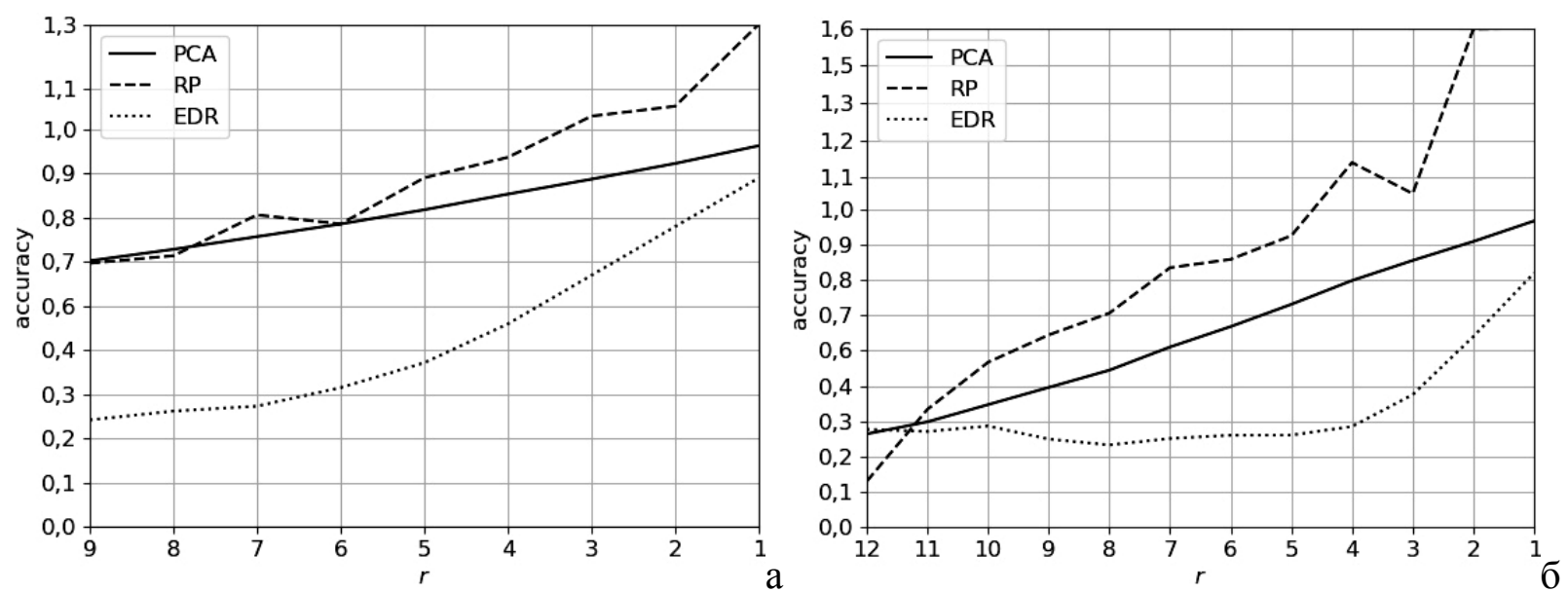

Рис.3. Относительные информационные потери для наборов данных simulation и heart.

Относительные информационные потери для EDR для различных значений $\delta$ приведены в табл.10-11.

Таблица 10. Зависимость $I(\delta)$ для метода EDR на наборе данных simulation.

\begin{tabular}{|c|c|c|c|c|c|}
\hline$\delta$ & 9 & 8 & 7 & 6 & 5 \\
\hline 0.1 & 0.0959 & 0.0997 & 0.1594 & 0.2795 & 0.3996 \\
\hline 0.2 & 0.2000 & 0.1932 & 0.1982 & 0.2656 & 0.3880 \\
\hline 0.3 & 0.2896 & 0.2896 & 0.2952 & 0.2943 & 0.3805 \\
\hline 0.4 & 0.3615 & 0.3887 & 0.3933 & 0.3981 & 0.3992 \\
\hline 0.5 & 0.3971 & 0.4842 & 0.4707 & 0.5130 & 0.4954 \\
\hline
\end{tabular}

Таблица 11. Зависимость $I(\delta)$ для метода EDR на наборе данных heart.

\begin{tabular}{|c|l|l|l|l|l|l|}
\hline$\delta \sim$ & 12 & 11 & 10 & 9 & 8 & 7 \\
\hline 0.1 & 0.1071 & 0.0478 & 0.0821 & 0.1244 & 0.1065 & 0.1254 \\
\hline 0.2 & 0.5947 & 0.0898 & 0.0676 & 0.0568 & 0.1435 & 0.1428 \\
\hline 0.3 & 0.3344 & 0.7126 & 0.6659 & 0.3530 & 0.2961 & 0.2992 \\
\hline 0.4 & 0.2921 & 0.3878 & 0.3833 & 0.4066 & 0.2814 & 0.3974 \\
\hline 0.5 & 0.2134 & 0.2683 & 0.3932 & 0.4452 & 0.4670 & 0.4310 \\
\hline
\end{tabular}


Графики указанных зависимостей показаны на рис.4.
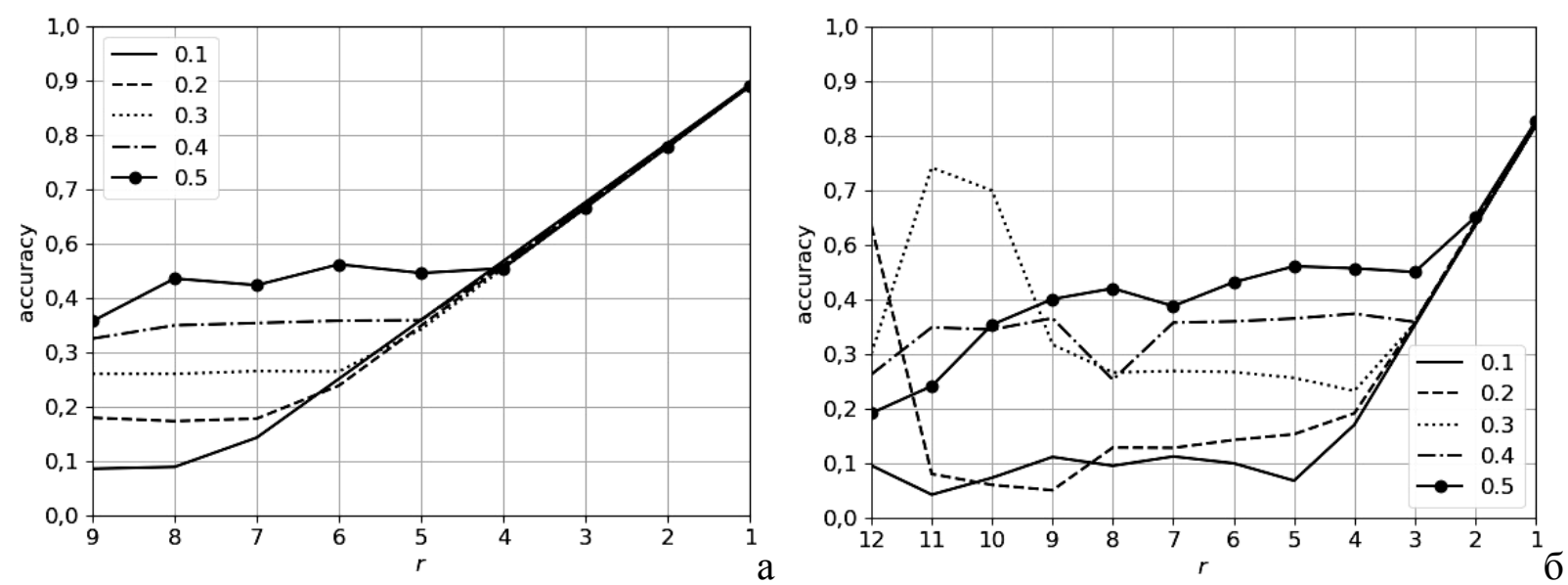

Рис.4. Зависимости $I(\delta)$ для EDR для наборов данных simulation и heart.

Проведенные исследования позволяют сделать следующие выводы.

- Метод EDR работоспособен при при любом объеме данных в обучающей и тестовой коллекциях данных; PCA не работоспособен при объемах данных меньше размерности признакового пространства; работоспособность RP требует дополнительного исследования;

- Уровень информационных потерь при применении метода EDR coxраняется до двухкратной редукции признакового пространства; для PСА и RP уровень информационных потерь существенно увеличивается с уменьшением размерности признакового пространства;

- Наличие в алгоритме метода EDR управляющего параметра $\delta$ позволяет регулировать уровень отностительных информационных потерь, тогда как в методах PCA и RP такая возможность отсутствует.

\section{7. Заключение}

Предлагается новый метод редукции признакового пространства (EDRметод), ориентированный на задачи машинного обучения для прогнозирования и классификации. Основу его составляет минимизация кросс-энтропийного «расстояния» по матрицам-проекторам с ограничениями по их фробениусовым нормам и информационной емкости. Развит алгоритм минимизации на базе мультипликативной схемы.

Исследована эффективность метода для задач бинарной классификации. Проведен вычислительный эксперимент по сравнению предлагаемого метода с методами главных компонент и случайных проекций редукции на двух наборах данных, показавший преимущества и особенности указанных методов. 
Кросс-энтропийная редукции матрищь данных с ограничением ...

\section{СПИСОК ЛИТЕРАТУРЫ}

1. Hastie Trevor, Tibshirani Robert, Friedman Jerome. The Elements of Statistical Learning: Data mining, Inference, and Prediction. - New York: Springer, 2009.

2. Воронцов К.В. Математические методы обучения по прецедентам / Курс лекций, МФТИ, 2013.

Vorontsov K.V. Matematicheskie metodi obuchenia po precedentam / Lecture Course, MIPT, 2013.

3. Van der Maaten Laurens, Postma Eric, Van den Herik Jaap. Dimensionality Reduction: A Comparative Review // TiCC TR, 2009, v.005, № 1, p.1-35.

4. Fodor I.K. A Survey of Dimension Reduction Techniques, 2002, № 1, p.1-18. Access mode: http://www.osti.gov/servlets/purl/15002155-mumfPN/native/.

5. Bruckstein A.M., Donoho D.L., Elad M. From sparse solutions of systems of equations to sparse modeling of signals and images // SIAM review, 2009, v.51, № 1, p.34-81.

6. Кендалл М., Стьюарт А., Гальчук Л.И., Терехин А.Т. Статистические методы и связи. - М.: Наука, 1973.

Kendall M., Stewart A., Galchuk L.I., Terekhin A.T. Statisticheskie methodi i sviazi. - M.: Nauka, 1973.

7. Jolliffe I.T. Principal component analysis. - New York: Springer-Verlag, 1986.

8. Comon P., Jutten C. Handbook of Blind Source Separation. Independent Component Analysis and Applications. - Oxford: Academic Press, 2010.

9. Berry M.W., Browne M. Algorithms and Applications for Approximate Nonnegative Matrix Factorization // Computational Statistics \& Data Analysis. 2007, v.52, p.155-173.

10. Поляк Б.Т., Хлебников М.В. Метод главных компонент: робастные версии // Автоматика и телемеханика, 2017, № 3, с.130-148;

Polyak B.T., Khlebnikov M.V. Principle component analysis: Robust versions // Automation \& Remote Control, 2017, v.78, p.490-506; https://doi.org/10.1134/ S0005117917030092.

11. Bingham Ella, Mannila Heikki. Random projection in dimensionality reduction: applications to image and text data // Proc. of the seventh ACM SIGKDD international conference on Knowledge discovery and data mining / ACM, 2001, p.245-250.

12. Vempala Santosh $S$. The random projection method // American Math. Soc., 2005, v.65.

13. Johnson W.B., Lindenstrauss J. Extensions of Lipshitz mapping into Hilbert Space // Modern Analysis and Probability / Amer. Math. Soc., 1984, v.26, p.189-206.

14. Achlioptas D. Database-friendly random projections // PODS'01 / Amer. Math. Soc., 2001, p.274-281.

15. Peng H.C., Long F., Ding C. Feature selection based on mutual information: criteria of max-dependency, max-relevance, and min-redundancy // IEEE Trans. on Pattern Analysis and Machine Intelligence, 2005, v.27, № 8, p.1236-1238.

16. Zhang Y., Li S., Wang T., Zhang Z.. Divergence-based feature selection for separate classes // Neurocomputing, 2013, v.101, p.32-42.

17. Попков Ю.С., Дубнов Ю.А., Попков А.Ю. Энтропийная редукция размерности в задачах рандомизированного машинного обучения // Автоматика и телемеханика, 2018, № 11, c.106-122; http://dx.doi.org/10.31857/S000523100002747-5

Popkov Y.S., Dubnov Y.A., Popkov A.Y. Dimension Reduction Method for Randomized Machine Learning Problems // Automation \& Remote Control, 2018, v.79, p.2038-2051. 
https:// doi.org/ 10.1134/ S0005117918110085

18. Magnus Jan R., Neudecker Heinz. Matrix differential calculus with applications in statistics and econometrics. Wiley, 1988.

19. Поляк Б.Т. Введение в оптимизацию. - М.: Наука, 1983;

Polyak B.T. Vvedenie v optimizaciu. - M.: Nauka, 1983.

20. Стрекаловский А.С. Элементы невыпуклой оптимизации. - Новосибирск: Наука, 2003. Strekalovskiy A.S. Elementy nevipukloy optimizacii. - Novosibirsk: Nauka, 2003.

21. Попков Ю.С. Теория макросистем. Равновесные модели. - M.: URSS, 2012;

Popkov Y.S. Teoria macrosistem. Ravnovesnie modeli. - M.: URSS, 2012.

22. Bishop C. Pattern Recognition and Machine Learning // Information Science and Statistics. - New York: Springer, 2007.( 1st edn. 2006. corr. 2nd printing edn.)

23. Friedman Jerome, Hastie Trevor, Tibshirani Robert. The elements of statistical learning // Springer series in statistics. - Berlin: Springer,2001, v.1.

24. Weinberger Kilian Q., Saul Lawrence K. Unsupervised learning of image manifolds by semidefinite programming // International J. of Comp.Vision. 2006, v.70, № 1, p.77-90. http://dx.doi.org/10.1007/s11263-005-4939-z

25. Saul L.K., Roweis S.T. Think globally, fit locally: unsupervised learning of low dimensional manifolds // Journal of Machine Learning Research, 2003, v.4, p.119-155.

26. Pedregosa F., Varoquaux G., Gramfort A. et al. Scikit-learn: Machine Learning in Python // Journal of Machine Learning Research, 2011, v.12, p.2825-2830.

27. Buitinck Lars, Louppe Gilles, Blondel Mathieu et al. API design for machine learning software: experiences from the scikit-learn project // ECML PKDD Workshop: Languages for Data Mining and Machine Learning, 2013, p.108-122.

28. KEEL Dataset repository. https://sci2s.ugr.es/keel/datasets.php. Accessed: 2019-07-03.

29. A software package for sequential quadratic programming: Rep.: DFVLR-FB 88-28 / DLR German Aerospace Center - Institute for Flight Mechanics; Executor: Dieter Kraft. Koln, Germany: 1988.

Поступила в редакцию 15.01.2020

После доработки 12.03.2020

Принята к публикации 20.04.2020 